\title{
Recent Advances in Hollow Fiber Technology for Telecoms Applications
}

\author{
E. Numkam Fokoua, G. T. Jasion, Y. Chen, S. R. Sandoghchi, T. D. Bradley, D. R. Gray, N. V. \\ Wheeler, J. R. Hayes, M. N. Petrovich, D. J. Richardson and F. Poletti. \\ Optoelectronics Research Centre, University of Southampton, Highfield, Southampton, SO17 1BJ, UK
}

\begin{abstract}
We review our recent work on the modelling, fabrication and characterization of hollow-core photonic bandgap fibers. We discuss the modal content of these fibers, as well as the opportunities and challenges presented by modal interactions in space division multiplexed transmission applications.
\end{abstract}

Over the past few years, improvements in the fabrication of hollow-core photonic bandgap fibers (HC-PBGFs) have led to seminal experiments demonstrating both single mode and mode division multiplexed data transmission in these fibers over a few to a few tens of $\mathrm{km}$ [1-4]. Key to these improvements in the fabrication process and the yield of a single fiber draw has been the development of numerical tools capable of predicting the evolution of the fiber structure during the draw, thereby bypassing the empirical trial and error approach $[5,6]$. Combining our fluid dynamics model with finite element mode-solving has allowed the prediction of the optical properties of fibers as a function of starting preform structures and draw parameters [7].

Although the lowest attenuation achieved in HC-PBGFs is still too high for long-haul data transmission [8, 9], the virtual elimination of optical nonlinearity and the near vacuum latencies offered by these fibers are increasingly important in a growing number of short reach data transmission applications. Achieving reasonably low attenuation levels in these fibers almost always requires the use of a core large enough to support multiple modes of propagation [10]. This presents the opportunity of using each of these modes as separate channels over which to encode data for transmission. To do so effectively requires a thorough characterization of the modal properties of the fibers, such as loss and group delay, and knowledge of the intermodal cross-talk. In these fibers intermodal cross-talk remains sufficiently low for mode division multiplexing over a few hundred meters of fiber, as confirmed by our earlier result demonstrating a record $73 \mathrm{~Tb} / \mathrm{s}$ capacity through transmission of $96 x 16 \mathrm{QAM}$ DWDM channels, launched over both polarizations of the 3 lowest spatial modes of a $310 \mathrm{~m}$ long 37 cell PBGF [2,3]. Routine characterization of the modal content of fabricated HC-PBGFs with 19 or 37 cell core defects using both spatially and spectrally resolved mode imaging $\left(\mathrm{S}^{2}\right)[11,12]$ and time of flight techniques reveal however that this level of intermodal cross-talk is significantly higher than in conventional solid fibers [13]. It is to be noted though, that despite this, selective excitation of the fundamental mode makes it possible to achieve single mode transmission over distances as long as $11 \mathrm{~km}$ of fiber [4].

Interestingly, like many of their other properties, intermodal cross-talk in HC-PBGFs lends itself to modification through appropriate design of the fiber structure. Our recently developed models show that since modal cross-talk is caused by the same longitudinal irregularities that give rise to loss, efforts in loss reduction simultaneously result in cross-talk reduction. We therefore predict that should the loss of hollow core fibers ever reach the level of conventional fibers, their intermodal cross-talk will be nearly two orders of magnitude lower, making them a potentially attractive technology for MIMO-less mode division multiplexing [13].

This talk will present some of our latest results in the development of long lengths of hollow core PBGFs suitable for high capacity data transmission, and our recent activities in the study of their differential modal properties.

\section{REFERENCES}

[1] F. Poletti et al., Nat. Photonics 7(4), pp.279-284 (2013)

[2] Y. Jung et al., Proc OFC'13, PDP5A.3

[3] V. A. J. M. Sleiffer et al., Proc OFC'13, OW1I.5

[4] Y. Chen et al, Proc OFC '15, Th5A.1 (Postdeadline)

[5] G.T. Jasion et al, Optics Express Vol.23(1) pp.312-329 (2015)

[6] G.T. Jasion et al, Optics Express 23(25) pp.32179-32190 (2015)

[7] G.T. Jasion et al, Proc ECOC'15 paper ID : 0395

[8] B. J. Mangan et al, Proc OFC'04, PDP24

[9] P. J. Roberts et al, Optics Express 13, pp. 236-244 (2005).

[10] R. F. Cregan et al., Science, 285, 1537, (1999)

[11] J. Nicholson et al, Optics Expres 16, pp. 7233 -7243 (2008)

[12] D. R. Gray et al, IEEE Photonics Technology Letters 28 (9), pp.1034-1037 (2016)

[13] E. Numkam et al, Proc. ECOC' 15, paper ID : 0664 